\title{
Diffuse Ultraviolet Background Radiation
}

\author{
Richard C. Henry ${ }^{1,2,3}$ \\ Henry A. Rowland Department of Physics and Astronomy, \\ The Johns Hopkins University, Baltimore, MD 21218-2686
}

\begin{abstract}
Diffuse ultraviolet background radiation may contain important information concerning the dark matter of the universe. I briefly review new Voyager observations of the diffuse background, which give a very low upper limit on the background radiation shortward of Lyman $\alpha$, and I review the capabilities for detection and characterization of diffuse radiation that will be provided by a proposed new NASA mission. Low-surface-brightness radiation remains largely an unexplored frontier, particularly in the ultraviolet.
\end{abstract}

\section{Introduction}

While most research in astronomy has focused on study of celestial point sources, the astronomers of the present IAU Colloquium consider, instead, the lowsurface-brightness universe: extended objects (and backgrounds) that most astronomers have by and large neglected. Diffuse backgrounds always contain independent information about the universe, in some cases of considerable significance: witness the famous $3 \mathrm{~K}$ background radiation.

O'Connell (1987) has pointed out the great importance of the ultraviolet spectral region as a hunting ground for low-surface-brightness radiation. $\mathrm{He}$ notes that in the visible, the much brighter zodiacal light renders such searchs difficult. This is the same point that was made by Disney (1976), who suggested that astronomers are blinded in the visible by the extraordinarily high sky background.

In Figure 1, I show the spectrum of the diffuse background radiation at high galactic latitudes, from the near infrared to the X-ray, including not only the ultraviolet, but also the extreme ultraviolet, where the interstellar medium is opaque.

The data that are presented in the figure indicate that there is a drop of $2 \frac{1}{2}$ orders of magnitude in the diffuse background between the visible diffuse background and the soft X-ray background. The region from $912 \AA$ to the soft X-ray is obscured by interstellar gas photoionization. One key question is,

\footnotetext{
${ }^{1}$ Director, Maryland Space Grant Consortium

${ }^{2}$ Principal Professional Staff, Applied Physics Laboratory, The Johns Hopkins University

${ }^{3}$ rch@pha.jhu.edu
} 


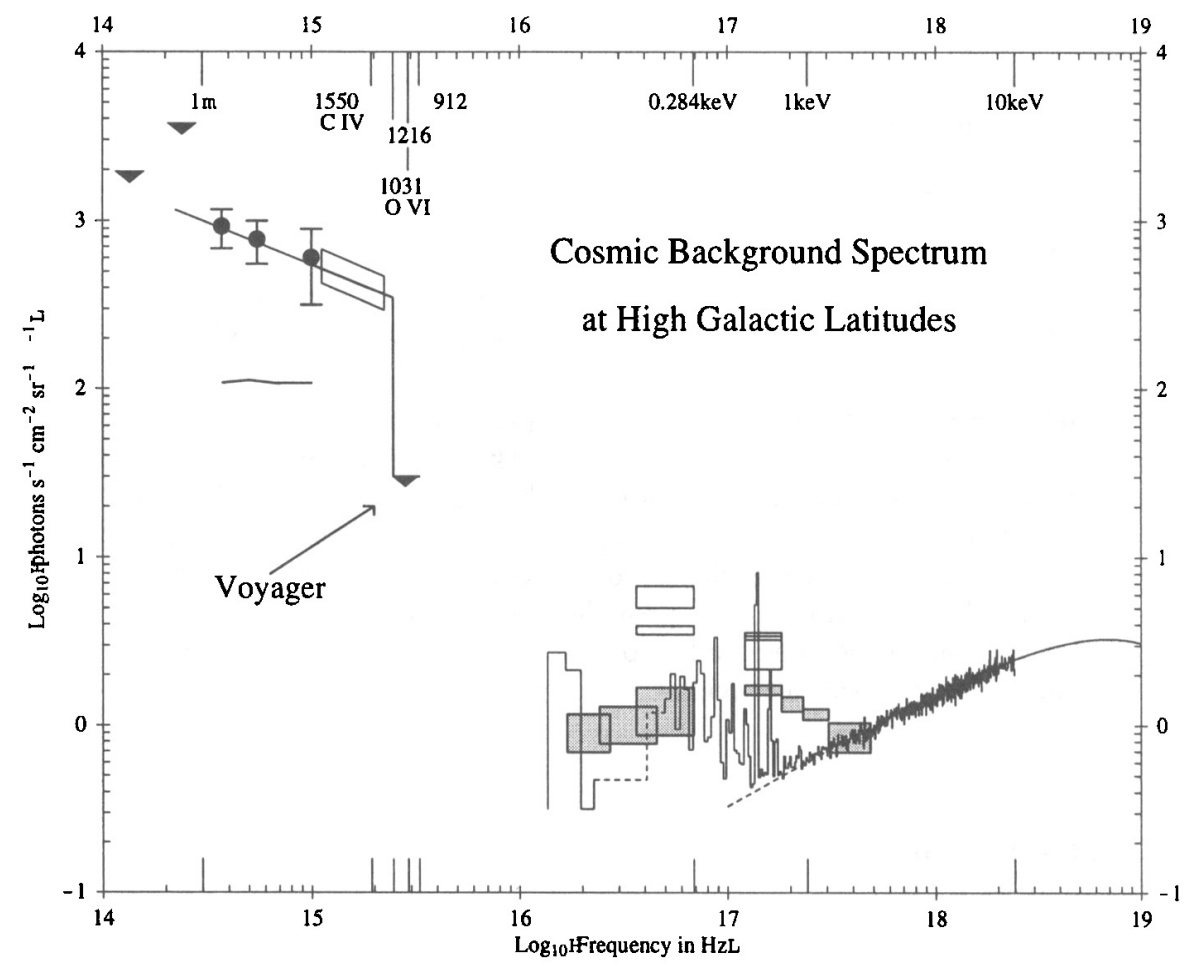

Figure 1. The black triangles are DIRBE upper limits (Hauser et al. 1998), while the three black circles are the HST optical background radiation data of Bernstein (1997); below the latter, the horizontal line shows her measure of the contribution (to that) from galaxies. Vogeley (1997) has shown that the excess is truly diffuse. The line through Bernstein's three points is the extrapolation of the model that was found by Henry \& Murthy (1995) to fit the UV background radiation measurements (box). The extraordinary drop to $\leq 30$ photons $\mathrm{cm}^{-2} \mathrm{~s}^{-1} \AA^{-1}$ ["photon units"] at $\sim 1000 \AA$ was discovered by Holberg (1986), using the Voyager ultraviolet spectrometer, and confirmed by Murthy, Henry, \& Holberg (1991) and Murthy et al. (1998). It has been interpreted by Henry (1991) as indicating that the high-galacticlatitude background, longward of Ly $\alpha$, is largely redshifted recombination radiation from an ionized intergalactic medium. In the X-ray part of the spectrum, I compare a HUBE-CUBIC (see text) simulated spectrum of the high-galactic-latitude CXRB [based on the rocket spectrum of Mendenhall \& Burrows (1998)], with the spectrum of Boldt (1987, solid line); and with the summary (shaded boxes) of McCammon \& Sanders (1990), and the early observations (open boxes) of Bowyer, Field, \& Mack (1968), Henry et al. (1968, 1971), and Davidsen et al. (1972). ROSAT's imaging has revealed an extremely complex soft X-ray sky, but even so, Wang \& McCray (1993) have suggested that radiation from a several-million-degree IGM may have been identified. 


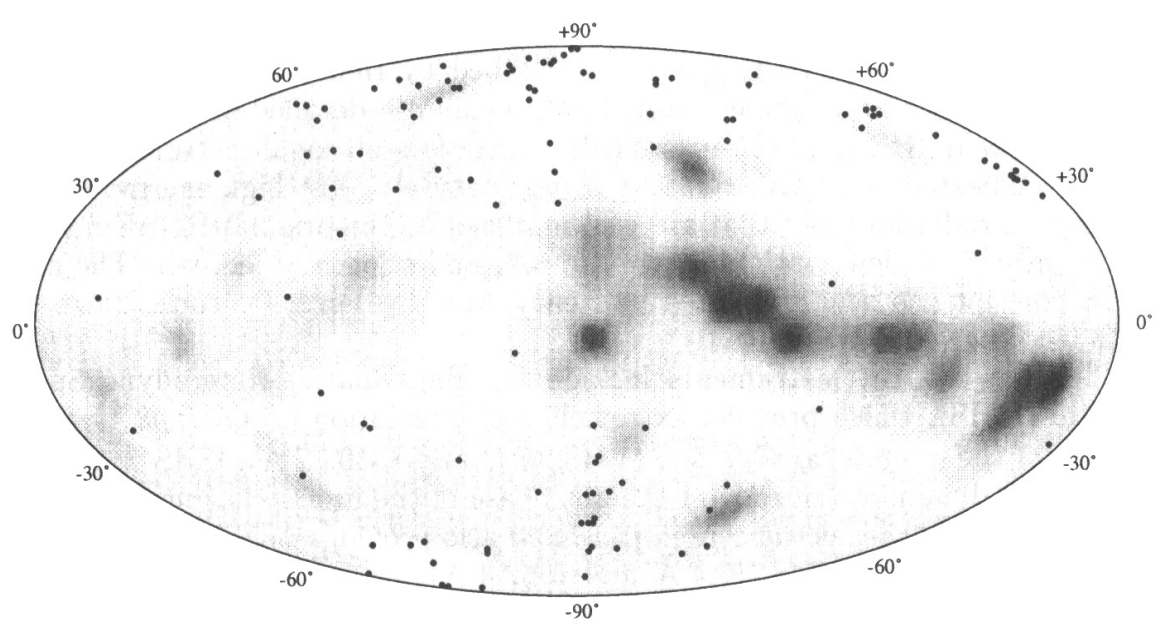

Figure 2. North is at the top, and the galactic center at the center, in this Aitoff map of the sky showing the new (Murthy et al. 1998) Voyager $(\sim 1000 \AA)$ limits (filled circles) on the diffuse ultraviolet background radiation. Of the 431 new observations, 265 (or $62 \%$ ) are $3 \sigma$ upper limits. The points plotted in the figure are measurements for which the sum of the measured flux and its error bar are less than 300 photon units. For the points plotted, the average measured flux is $24.5 \pm 8.0$ photon units. The shaded background is an integration of the light at $1565 \AA$ that is provided by direct radiation from TD1 stars. This radiation can scatter from interstellar dust, creating a diffuse background (Bowyer 1991). Note the presence of Gould's Belt, delineated by hot stars. Gould's Belt is a segment of the galaxy that is tipped $19^{\circ}$ relative to the galactic plane.

where precisely does the large drop in background occur? I believe it occurs at $1216 \AA$, and that the longer wavelength radiation that is observed at high galactic latitudes is largely redshifted Lyman $\alpha$ radiation. If that view should turn out to be correct, then longward of $1216 \AA$ we are seeing recombination radiation from an ionized intergalactic medium.

The exciting ideas concerning dark matter in the universe that are suggested by the data of Figure 1 are in part predicated on the reality of the sharp drop in the diffuse ultraviolet background radiation at $1216 \AA$ that the Voyager data suggest exists. In Figure 2, I show the distribution on the sky of the new Voyager upper limits of Murthy et al. (1998), one example of which is shown in Figure 3 (a). While there are, now, a very large number of such upper limit points, the importance of the result demands a dedicated confirmation investigation.

I have proposed such an investigation, as one part of Hot Universe Background Explorer (HUBE), a proposal which was submitted to NASA in 1998 August as a candidate MIDEX mission. HUBE is an improved version of the 
HUBE proposal that was accepted by NASA in 1996 as a MIDEX Alternate Mission. The original HUBE has been described by Henry $(1997,1998)$, while the history of my search for the intergalactic medium is detailed in Henry (1995).

The new HUBE has been expanded to include additional instruments that are also dedicated to the elucidation of the nature of the high-energy diffuse background radiation, and that are well matched to the original HUBE instruments: in field of view, optimum target dwell, and science objectives. The new HUBE concept has been presented by Henry, Murthy, Ford, Peacock, Burrows, Smith, \& Bloch (1998).

The new HUBE instruments include Imaging Spatial Heterodyne Spectrometer (ISHS), which provides extremely high resolution $(\Delta \lambda=0.08 \AA)$ spectroscopy of the interstellar CIV line (1548.20 $\AA$ and $1550.77 \AA$ ). ISHS will allow study of the dynamics not only of the hot phase of the interstellar medium, but also of the cold phase, as the chosen passband also provides velocity information on two $\mathrm{H}_{2}$ emission lines (1547.4 $\AA$ and $1562.4 \AA$ ).

A second new instrument is "Cosmic Unresolved Background Instrument using CCD's" (CUBIC), which has been flown previously, on the ill-fated Argentine SAC-B mission. CUBIC will provide high-sensitivity, excellent-resolution spectroscopy of the diffuse background radiation from $1.24 \AA$ to $250 \AA$. With CUBIC, too, there is hope of addressing the question of an intergalactic medium (see Figure 1).

\section{Neutrino Decay Radiation}

The final new instrument in the HUBE complement is a spectrometer that straddles Lyman $\alpha$, permitting detection of a step at Lyman $\alpha$ with a single spectrometer (as opposed to separate spectrometers above and below Lyman $\alpha$ ). A simulation of the result of an observation with this new spectrometer at high galactic latitudes is presented in Figure 3 (b), on the assumption that the observed drop at Lyman $\alpha$ is real. The new spectrometer has been specifically designed to reject terrestrial/solar-sytem Lyman $\alpha$ radiation. The spectrometer is double-pass, and on the first pass, Lyman $\alpha$ is blocked. This means that only doubly-scattered Lyman $\alpha$ can reach the wrong part of the spectrum, and with modern gratings, this amount of radiation is totally negligible. Thus, if the break at Lyman $\alpha$ is real, this spectrometer (designated $L \alpha S$ ) will establish that fact unequivocally.

If the diffuse ultraviolet background at high galactic latitudes is truly redshifted Lyman $\alpha$ radiation, the intensity is such that to explain the radiation, not only must there be an intergalactic medium containing all of the missing baryonic dark matter, but that medium must be so clumped that it would have already recombined, unless some strong source of ionizing radiation is present beyond that which is provided by quasars and galaxies. Sciama (1998) has suggested just such an ionizing radiation source, namely, massive neutrinos that decay with the emission of an ionizing photon. Our Voyager observations can be taken to imply that Sciama's suggestion is correct.

A direct search for the ionizing photons themselves is underway, the EURD detector aboard the Spanish MINISAT 01 satellite, but in Figure 4 we show that this mission is likely to fail, due to the interfering effects of terrestrial airglow. 

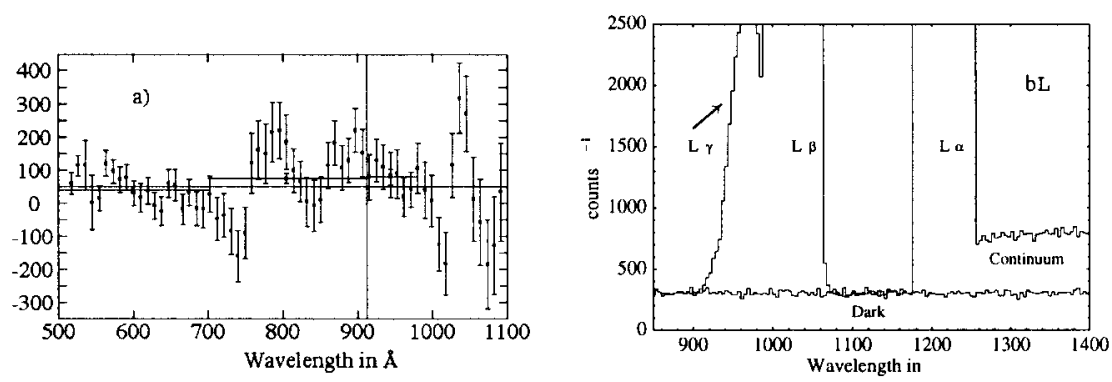

Figure $3 . \quad$ a) Example of a Voyager spectrum of the diffuse ultraviolet background at high galactic latitude (Murthy et al. 1998). The data are corrected so that no signal appears below the interstellar hydrogen photoionization edge at $912 \AA$, as is expected. It can be seen that there is no detection of diffuse radiation longward of this wavelength either. b) Simulation of an observation at high galactic latitudes of the cosmic diffuse ultraviolet background radiation, with our spectrometer which straddles the intense geocoronal/solar-system Lyman $\alpha$ radiation. The spectrometer is double-pass, allowing strong rejection of (blocked) Lyman $\alpha$. The resolution is $40 \AA$. Longward of Lyman $\alpha$, a continuum of extragalactic radiation is seen, but that continuum does not continue below Lyman $\alpha$, indicating that it is surely made up of red-shifted Lyman $\alpha$ radiation. Strong terrestrial/solar-system Lyman $\beta$ and Lyman $\gamma$ radiation are also seen. No continuum is expected below $912 \AA$ in any model of the origin of the high-latitude diffuse UV background. That there actually does exist an extragalactic diffuse ultraviolet background (longward of $1216 \AA$ ), of $300 \pm 80$ photon units, is supported by Witt \& Pettersohn (1994).
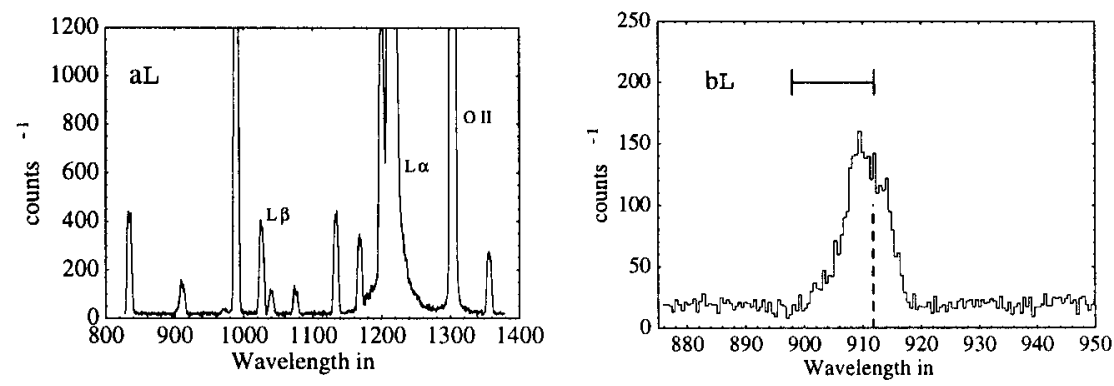

Figure 4 a) The day airglow observed by the Hopkins Ultraviolet Telescope (HUT) between $800 \AA$ and $1400 \AA$. b) A magnified view, highlighting the critical region, near the Lyman limit (dashed line), where Sciama (1998) has predicted emission from decaying neutrinos (the horizontal error bar shows the range that is permitted by Sciama's theory). The interested reader should consult Feldman et al. (1992) for examples showing HUT spectra of the night airglow, which demonstrate the strong time/place variation of the airglow complex around $910 \AA$. 

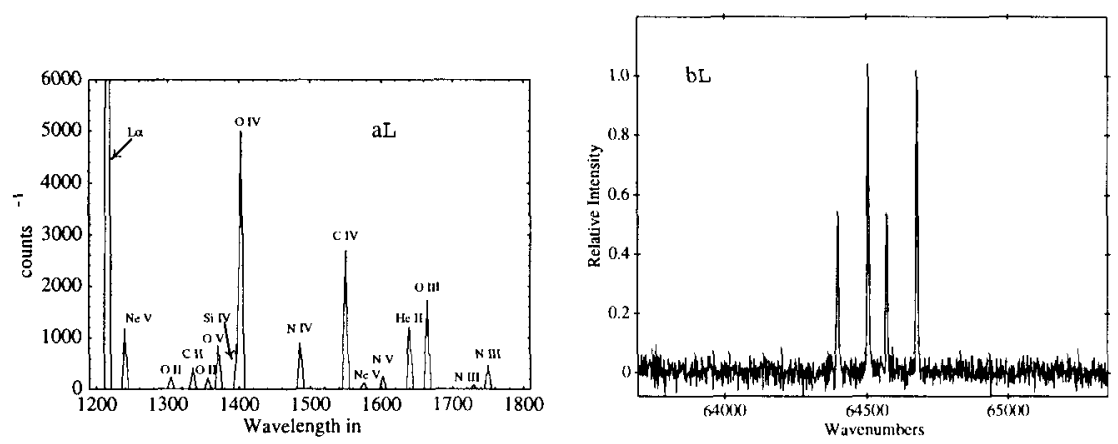

Figure 5. a) A one-hour exposure with HUBE-FUVS of a 1.5-arcminute by 2.7-arc-minute section of the Cygnus Loop nebula, showing, for example, strong CIV emission. The HUBE instruments will be used to make a detailed map of these emissions over the entire Cygnus Loop nebula. b) Simultaneously, HUBE-ISHS obtains a very-highresolution spectrum of the CIV emission, not only resolving the two components, but, thanks to Doppler shifts, separating the emission from the side of the shell that is nearest to us, from the emission from the portion of the shell that is farthest from us. (This simulation was created by Jeffrey J. Bloch, Barham W. Smith, and John M. Harlander.)

\section{HUBE and the Cygnus Loop}

The power of the new version of HUBE in studying diffuse radiation can be shown most clearly by displaying what HUBE might accomplish in the examination of a relatively bright source of diffuse ultraviolet and X-radiation, the Cygnus Loop nebula. In Figure 5, I show simulations of observation of the Cygnus Loop with our Far Ultraviolet Spectrometer (HUBE-FUVS), and with our Imaging Spatial Heterodyne Spectrometer (HUBE-ISHS). ISHS co-inventors are HUBE Co-Investigators John Harlander and Fred Roesler (Harlander and Roesler 1990).

In Figure 6, I show observations of the Cygnus Loop (again simulations, of course) with our Extreme Ultraviolet Spectrometer (HUBE-EUVS), and with our X-ray Spectrometer (HUBE-CUBIC). CUBIC is the creation of Dr. David Burrows (Burrows 1996).

So, the HUBE complex of instruments can be seen to provide powerful diagnostics for celestial hot plasmas! In addition, HUBE offers strong synergism with the FUSE, HST, and GALEX missions. FUSE observes OVI in absorption; HUBE observes OVI in emission; together, they determine the filling factor. The same is true for molecular hydrogen fluorescence. GALEX, because it uses a grism, can carry out no spectroscopy of the diffuse background radiation, and (like HST) has no capacity of any kind below Lyman Alpha. Only our imager. HUBE-UVI, overlaps with GALEX: but HUBE-UVI, at f/1.7, has 15 times the diffuse-background sensitivity of f/6 GALEX (which is optimized to detect point sources against the diffuse background). It is, of course, our hope that NASA 

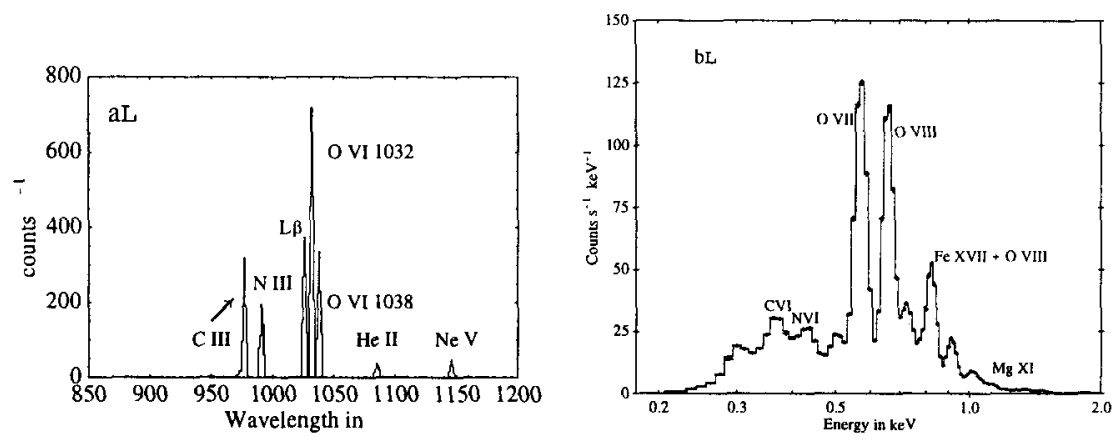

Figure 6. a) A simulated one-hour exposure with HUBE-EUVS of a 1.5-arc-minute by 2.7-arc-minute section of the Cygnus Loop nebula, showing strong OVI emission, with the two components resolved. Note that terrestrial Lyman $\beta$ emission is small compared with the nebular emission, and that the $3 \AA$ resolution of EUVS is enough to clearly separate OVI from Lyman $\beta$. b) Simultaneously, HUBE-CUBIC obtains a soft $\mathrm{X}$-ray spectrum of the Cygnus Loop. (This simulation was created by David N. Burrows.)

will select HUBE for implementation as the next Astrophysics MIDEX mission: in 1998 December, NASA is scheduled to pick two MIDEX finalists, and in mid-1999 to select, from these, a single mission for implementation.

Acknowledgments. I am grateful to the HUBE science team: Richard D. Belian, Luciana Bianchi, Jeffrey J. Bloch, Joel Bregman, David N. Burrows, Renyue Cen, Philip A. Charles, Jane Charlton, Mark Clampin, Eric Conway, Donald P. Cox, Laura Danly, Richard J. Edgar, Eric Feigelson, Holland Ford, Ernest Hammond, Gordon P. Garmire, John M. Harlander, Andrew D. Holland, Peter Jakobsen, Randy A. Kimble, Jeffrey Kruk, Dan McCammon, Jayant Murthy, David A. Neufeld, John H. Nousek, Robert W. O'Connell, Larry J. Paxton, Keith Peacock, William C. Priedhorsky, John C. Raymond, Ronald J. Reynolds, Fred L. Roesler, Diane Roussel-Dupré, Robin Shelton, Mark Skinner, Oswald H. W. Siegmund, Barham W. Smith, Stefan Thonnard, Martin J. Ward, Robert S. Warwick, and Richard Willingale. I am also most grateful for the support of the US National Aeronautics and Space Administration (NASA)!

\section{References}

Bernstein, R. A. 1997, PhD Thesis, CalTech

Boldt, E. 1987, Physics Reports, 146, 215

Bowyer, C. S. 1991, ARAA, 29, 59

Bowyer, C. S., Field, G. B., \& Mack, J. E. 1968, Nature, 217, 32

Burrows, D. N. 1996, CUBIC Instrument Handbook, PSU (publication available from http://www.astro.psu.edu/xray/cubic/papers/handbook/) 
Davidsen, A. F., Shulman, S., Fritz, G., Meekins, J. F., Henry, R. C., \& Friedman, H. 1972, ApJ, 177, 629

Disney, M. J. 1976, Nature, 263, 573

Feldman, P. D., Davidsen, A. F., Blair, W. P., Bowers, C. W., Durrance, S. T., Kriss, G. A., Ferguson, H. C., Kimble, R. A., \& Long, K. S. 1992, Geophys. Res. Lett., 19, 453

Harlander, J. M., \& Roesler, F. L. 1990, Proc. SPIE, 1235, 622

Hauser, M. G., Arendt, R. G., Kelsall, T., Dwek, E., Odegard, N., Weiland, J. L., Freudenreich, H. T., Reach, W. T., Silverberg, R. F., Moselely, S. H., Pei, Y. C., Lubin, P., Mather, J. C., Shafer, R. A., Smoot, G. F., Weiss, R., Wilkinson, D. T., \& Wright, W. L. 1998, ApJ (Letters), submitted

Henry, R. C., Murthy, J., Ford, H., Peacock, K., Burrows, D. N., Smith, B. W., \& Bloch, J. J. 1998, BAAS, 30, 926

Henry, R. C. 1998, in Multifrequency Behaviour of High Energy Cosmic Sources, F. Giovannelli \& L. Sabau-Graziati (eds.), Mem. S.A.It. Vol. 69 (in press)

Henry, R. C. 1997, in Advances in Stellar Evolution, ed. R. T. Rood \& A. Renzini, Cambridge Contemporary Astrophysics (Cambridge: CUP), p. 337

Henry, R. C. 1995, in The Physics of the Interstellar Medium and Intergalactic Medium, ASP Conference Proceedings, 80, ed. A. Ferrara, C. Heiles, C. McKee, \& P. Shapiro, (San Francisco: ASP), p. 561

Henry, R. C. 1991, ARAA, 29, 89

Henry, R. C., Fritz, G., Meekins, J. F., Friedman, H., \& Byram, E. T. 1968, ApJ (Letters), 153, 11

Henry, R. C., Fritz, G., Meekins, J. F., Chubb, T., \& Friedman, H. 1971, ApJ (Letters), 163, 73

Henry, R. C., \& Murthy, J. 1995, in Extragalactic Background Radiation, ed. D. Calzetti, M. Fall, M. Livio, \& P. Madau, (Cambridge: Cambridge University Press), 51

Holberg, J. B. 1986, ApJ, 311, 969

McCammon, D., \& Sanders, W. T. 1990, ARAA, 28, 657

Mendenhall, J. A., \& Burrows, D. N. 1998, in preparation

Murthy, J., Hall, D., Earl, M., Henry, R. C., \& Holberg, J. B. 1998, ApJ, submitted

Murthy, J., Henry, R. C., \& Holberg, J. B. 1991, ApJ, 383, 198

O'Connell, R. W. 1987, AJ, 94, 876

Sciama, D. W. 1998, A\&A, 335, 12

Vogeley, M. S. 1997, BAAS, 29, 1207; and ApJ, submitted

Wang, Q. D., \& McCray, R. 1993, ApJ (Letters), 409, 37

Witt, A. N., \& Petersohn, J. K. 1994, in ASP Conference Series 58, The First Symposium on the Infrared Cirrus and Diffuse Interstellar Clouds, ed. R. M. Cutri \& W. B. Latter (San Francisco: ASP), 91 